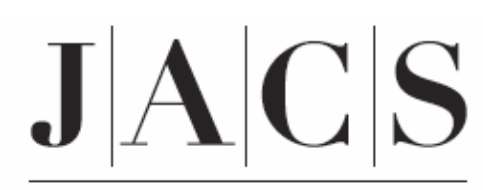

A R T I C L E S

\title{
Design and Optimization of Molecular Nanovalves Based on Redox- Switchable Bistable Rotaxanes
}

Thoi D. Nguyen, Yi Liu, Sourav Saha, Ken C.-F. Leung, J. Fraser Stoddart, ${ }^{*}$ and Jeffrey I. Zink*

Contribution from the California NanoSystems Institute and Department of Chemistry and Biochemistry, University of California, Los Angeles, 405 Hilgard Avenue, Los Angeles, California 90095-1569.

\section{Supporting Information}

\author{
REVISED VERSION
}

\begin{tabular}{|ll|}
\hline \multicolumn{2}{|c|}{$*$ Correspondence } \\
\hline Professor J Fraser Stoddart & Professes \\
California NanoSystems Institute and & California NanoSystems Institute and \\
Department of Chemistry and & Department of Chemistry and \\
Biochemistry, University of California, & Biochemistry, University of California, \\
Los Angeles, 405 Hilgard Avenue, Los & Los Angeles, 405 Hilgard Avenue, Los \\
Angeles, CA 90095-1569 (USA) & Angeles, CA 90095-1569 (USA) \\
Tel: (+1)-310-206-7078 & Tel: (+1)-310-825-1001 \\
Fax: (+1)-310-206-1843 & Fax : (+1)-310-206-4038 \\
Email: stoddart@chem.ucla.edu & Email: zink@chem.ucla.edu \\
& \\
\hline
\end{tabular}


Discussion of Figure S1. Reflectance infrared spectroscopy was used to characterize the mesoporous silica material after allyltriethoxysilane derivatization. The bending and the stretching modes associated with the allyl functional group of the allyltriethoxysilane linker are observed in both the $\mathrm{IN}_{\mathrm{CoSE}}\left(\mathrm{CH}_{2}\right.$ bend, $1490 \mathrm{~cm}^{-1}$; $\mathrm{C}=\mathrm{C}$ stretch, $\left.1660 \mathrm{~cm}^{-1}\right)$ and OUT $\left(\mathrm{CH}_{2}\right.$ bend, $1420 \mathrm{~cm}^{-1}$; C=C stretch, $\left.1640 \mathrm{~cm}^{-1}\right)$ locations. The permanent presence of allyltriethoxysilane, even after extensive washes, indicates that the allyltriethoxysilane linker reacted and formed a permanent bond with the silica framework. After solvent extraction in which a small amount of acid was used, the presence of the $\mathrm{C}=\mathrm{C}$ stretching mode shows that the alkene functionality was still present for subsequent [2]rotaxane derivatization.

A

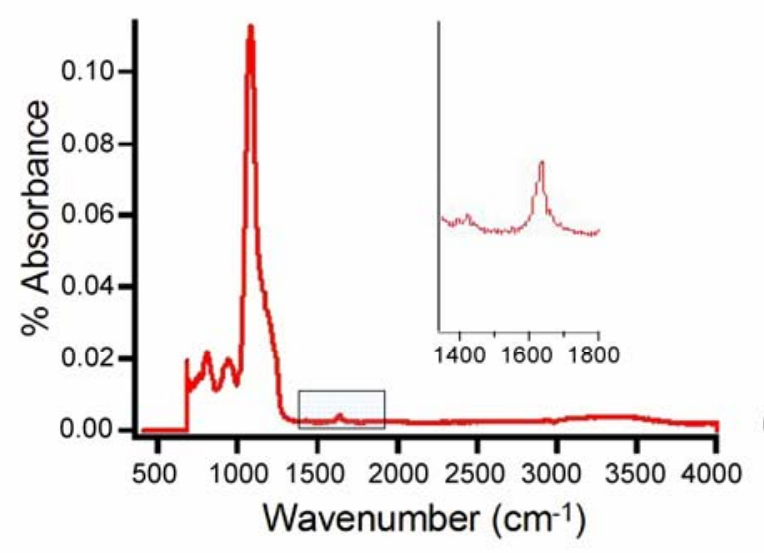

B

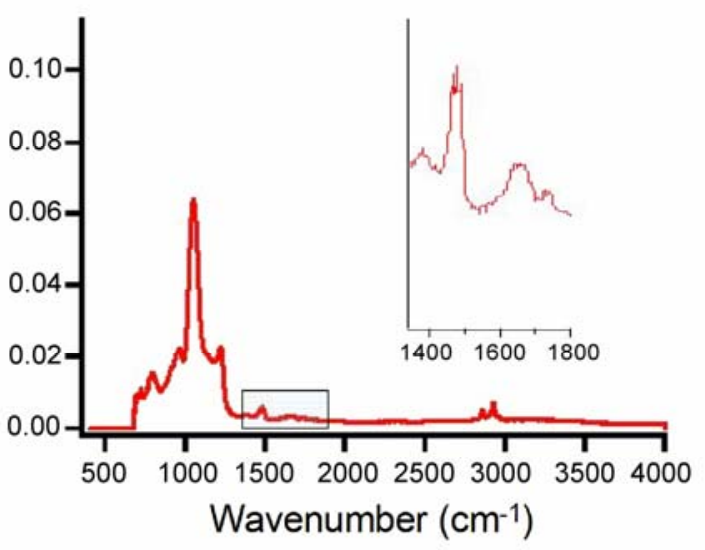

Figure S1. Reflectance infrared spectra for (A) b-OUT and (B) b-IN $\mathbf{b}$ Cose with the insets showing the zoom in regions.

Discussion of Figure S2. The $\mathrm{N}_{2}$ adsorption/desorption isotherms for the surfactantremoved MCM-41, allyltriethoxysilane linker-modified b-OUT, b-IN $\mathrm{S}_{\mathrm{SeD}}$, and $\mathbf{b}$-IN $\mathrm{IN}_{\mathrm{CoSe}}$ are shown in Figure S2. All samples show type IV isotherms, characteristic of mesoporous material according to IUPAC convention. For the $\mathrm{IN}_{\mathrm{SeD}}$ and $\mathrm{IN}_{\mathrm{CoSe}}$ 
locations, virtually no hysteretic loop was observed. A small amount of hysteresis was observed for surfactant-removed MCM-41 and the b-OUT. The long plateau in all samples at higher relative pressure indicates that pore filling is restricted to the inflection point at $p / p_{o}=0.18-0.29$. Compared to samples from which the surfactant has not been removed, all samples in Figure S2 exhibit a much higher degree of porosity, attesting to the high accessibility of the system when the surfactant has been removed. ${ }^{1}$ The lack of an $\mathrm{N}_{2}$ condensation curve in samples with surfactant still present, also indicates strongly that, since the pore is filled, chemical penetration into the pore is not viable. The large difference between the surfactant-filled and the surfactant-removed sample also indicates that the $\mathrm{N}_{2}$ condensation curve is associated with the condensation of $\mathrm{N}_{2}$ into the mesopores instead of into other material surfaces, a claim that has been substantiated to a great extent.
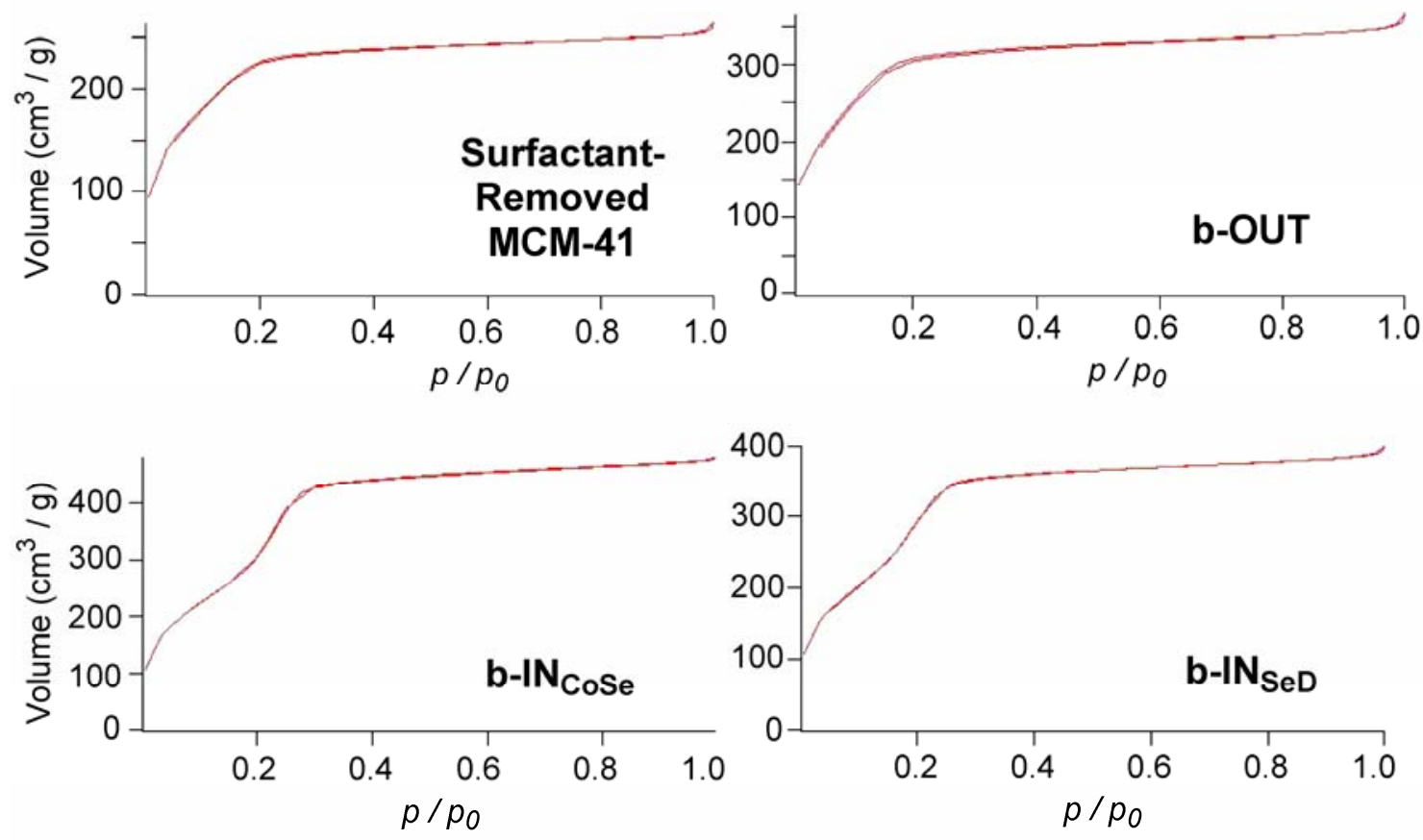

Figure S2. Nitrogen absorption/desorption isotherm of the surfactant-removed MCM-41, the allyltriethoxysilane linker-modified $\mathbf{b}$-IN $\mathrm{CoSe}_{\mathrm{C}} \mathbf{b}$-IN $\mathrm{INeD}_{\mathrm{Se}}$, and $\mathbf{b}$-OUT. 
Discussion of Figure S3. Upon gas-phase reaction to deposit the allyltriethoxysilane linker, the $\mathrm{IN}_{\mathrm{CoSe}}$ and the $\mathrm{IN}_{\mathrm{SeD}}$ locations show substantial difference from that of surfactant-removed MCM-41. For the $\mathrm{IN}_{\mathrm{SeD}}$ and the $\mathrm{IN}_{\text {CoSe }}$ locations, a second $\mathrm{N}_{2}$ condensation profile is observed. Since $\mathrm{N}_{2}$ condensation has been established to be capillary condensation of $\mathrm{N}_{2}$ inside the pore, this second $\mathrm{N}_{2}$ condensation profile can be thought of as a result of linker deposited inside the pores creating two different condensation profiles. In contrast to the IN locations, in the OUT location, a single condensation profile is observed (similar to surfactant-removed MCM-41, but with higher $\mathrm{N}_{2}$ volume adsorbed, indicating that the allyl linker provides more van der Waals' surface for $\mathrm{N}_{2}$ to adsorb). This lack of a secondary condensation provides strong evidence for the OUT location, since the linker can only be deposited outside and so cannot cause a second condensation. In the $\mathrm{IN}_{\mathrm{SeD}}$ and the $\mathrm{IN}_{\mathrm{CoSe}}$ locations, the lack of hysteretic curves indicates a phenomenon that results from the instability of the liquid $\mathrm{N}_{2}$ meniscus inside the narrow channel. ${ }^{2}$ The presence of weak hysteretic curves in the surfactant-removed MCM-41 and OUT locations indicates that these pores have a slightly enlarged dimension, relative to those of $I \mathrm{IN}_{\mathrm{SeD}}$ and the $\mathrm{IN}_{\mathrm{Cose}}$ locations. The shifting of the inflection points between the $\mathrm{IN}_{\mathrm{SeD}}$ and the $\mathrm{IN}_{\mathrm{CoSe}}$ locations, relative to the OUT location, also strongly supports this belief. Since linkers were deposited inside the pores in the IN locations, the linkers provide more van der Waals's surface for the $\mathrm{N}_{2}$ to condense on, resulting in a higher volume adsorbed; at the same time, however, there is a shift in the relative pressure to higher pressure on account of more steric hindrance. In the $\mathrm{IN}_{\mathrm{CoSe}}$ location, more linkers were deposited, resulting in a higher volume adsorbed and a higher pressure inflection point. Evidently, porosimetry via $\mathrm{N}_{2}$ isotherm can 
provide a mean to differentiate the two locations. The data also signify that, although the $\mathrm{IN}_{\mathrm{SeD}}$ and OUT locations are much more synthetically similar (the alternating of synthetic steps: solvent extraction and vapor phase deposition of linker), the $\mathrm{IN}_{\mathrm{SeD}}$ is much closer to the $\mathrm{IN}_{\mathrm{CoSe}}$ locations in term of the relative position of the deposited allyl linker. These observations also draw attention to the fact that, since allyltriethoxysilane in the OUT location does not hinder the pore, it forms the basis for a material with a much higher loading capacity. For the purpose of comparison, all sample exhibit similar d-spacings, as indicated by powder XRD.
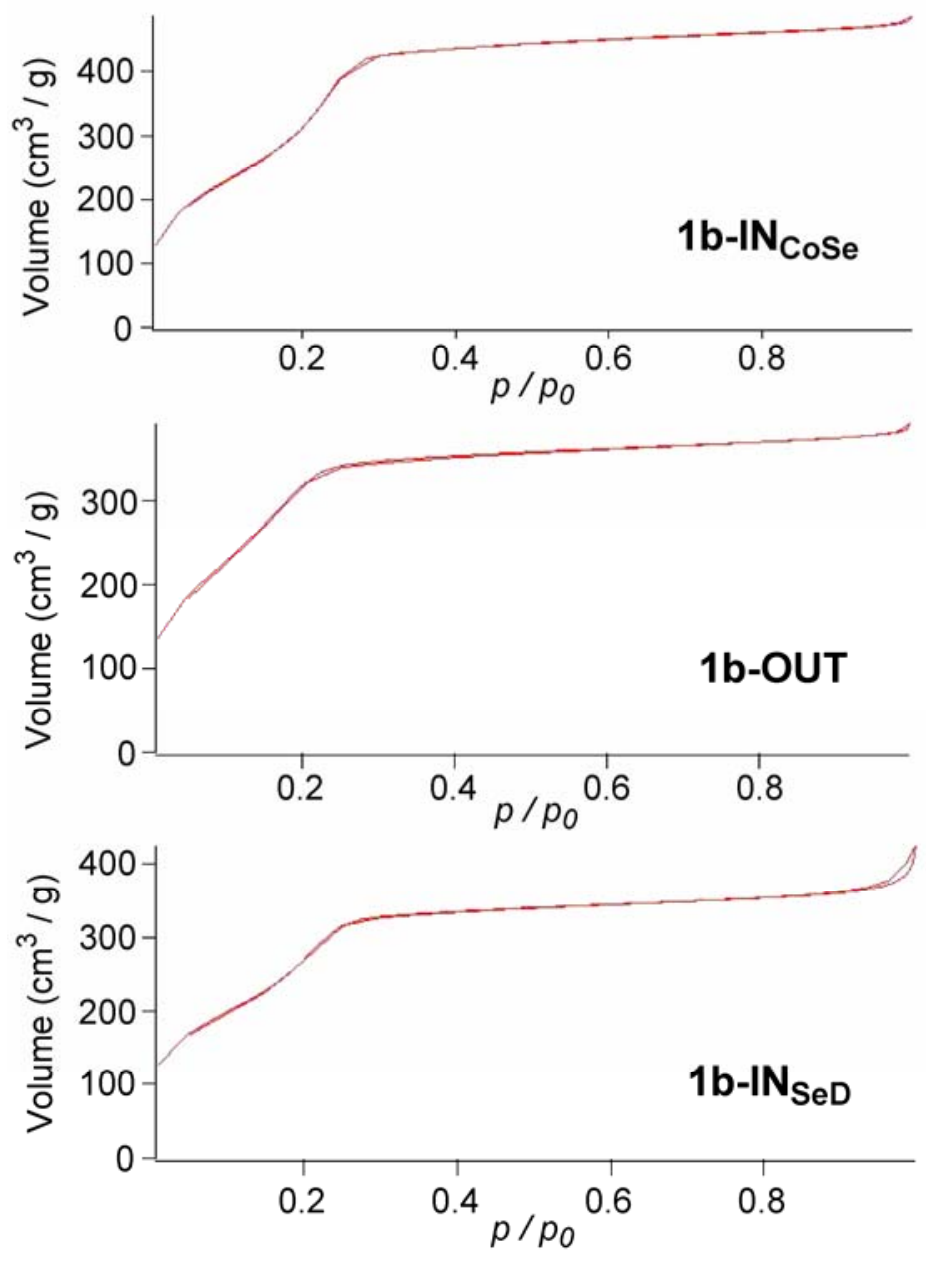

Figure S3. Nitrogen absorption/desorption isotherms of the [2]rotaxane (1)-terminated $\mathbf{1 b}-\mathrm{IN}_{\mathrm{CoSe}}, \mathbf{1 b}-\mathrm{OUT}$ and $\mathbf{1 b}-\mathrm{IN}_{\mathrm{SeD}}$. 
After the attachment with [2]rotaxane $\mathbf{1}^{\mathbf{4}^{+}}$to the linker-modified MCM-41, all three locations remain similar to that in their respective linker-derivatized location. The $\mathrm{N}_{2}$ isotherm of the nanovalves 1b-OUT exhibits (Figure S3) a faintly observable secondary $\mathrm{N}_{2}$ condensation and the inflection point reveals a minute shift to higher pressure, suggesting that the large [2]rotaxane can exert a considerable amount of hindrance in the pore.

Discussion of Figure S4. X-Ray diffraction was used to verify that modification of the MCM-41 materials with linkers in different configurations did not destroy the long-range order. The powder XRD patterns of the $\mathrm{IN}_{\mathrm{CoSE}}$ and OUT materials are shown in Figure S4. The peaks correspond to lattice spacings of $3.4 \mathrm{~nm}$ and $3.3 \mathrm{~nm}$, respectively. Calcination causes a small shift to higher angle (i.e. a small amount of shrinkage), but solvent extraction does not cause a shift.

(a)

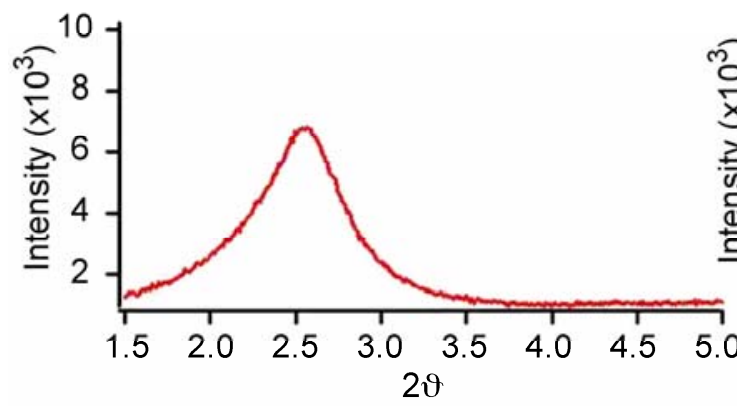

(b)

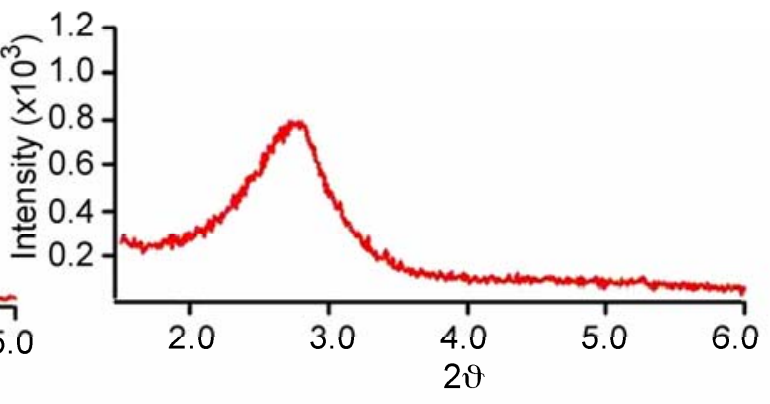

Figure S4. XRD Patterns of the surfactant-removed MCM-41 porous silica particles: (a) material with the linker in the $\mathrm{IN}_{\mathrm{CoSE}}$ location and (b) material with the linker in the OUT location. 
Discussion of Figure S5. Attachment of the [2]rotaxane to the the MCM-41 particles is monitored by fluorescence spectroscopy. The luminescence spectrum of the rotaxanederivatized MCM-41 particles is shown superimposed on the spectrum of the rotaxane dissolved in acetonitrile in Figure S5. The spectrum of the rotaxane on the derivatized particles is slightly broader than that of the rotaxane in solution but the peak maxima are observed at the same wavelength. The partial quenching of the luminescence of the DNP luminophore when the $\mathrm{CBPQT}^{4+}$ ring is associated with the DNP was used to monitor the operation of the valve (Figure 3 in the paper).

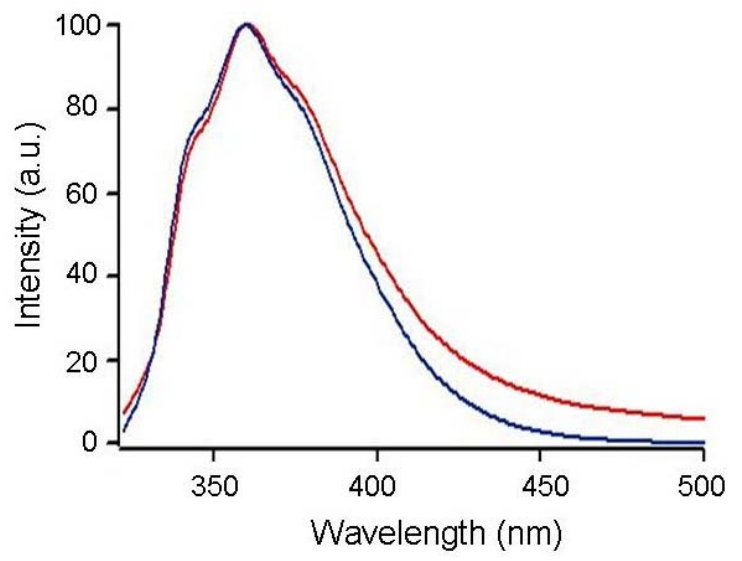

Figure S5. Fluorescence spectra of the bistable [2]rotaxane $\mathbf{1}^{\mathbf{4}}$ in MeCN (blue trace) and $\mathbf{1}^{4+}$ tethered to MCM-41 (red trace).

\section{References and Footnotes}

1. The tested coumarin was dissolved in MeCN and subjected to both ascorbic acid and $\mathrm{Fe}\left(\mathrm{ClO}_{4}\right)_{3}$. The luminescence spectra of coumarin before and after the addition of these agents were then compared with each other. No changes in the coumarin luminescence intensity nor shifts of the luminescence peak were observed.

2. Lim, M. H.; Stein, A. Chem. Mater. 1999, 11, 3285-3295. 


\section{Complete ref 3d from the article:}

3. (d) Azov, V. A.; Beeby, A.; Cacciarini, M.; Cheetham, A. G.; Diederich, F.; Frei, M.; Gimzewski, J. K.; Gramlich, V.; Hecht, B.; Jaun, B.; Latychevskaia, T.; Lieb, A.; Lill, Y.; Marotti, F.; Schlegel, A.; Schlittler, R. R.; Skinner, P. J.; Seiler, P.; Yamakoshi, Y. Adv. Funct. Mater. 2006, 16, 147-156. 